\title{
A Methodology for Implementing Highly Concurrent Data Objects
}

\author{
MAURICE HERLIHY \\ Digital Equipment Corporation
}

\begin{abstract}
A concurrent object is a data structure shared by concurrent processes. Conventional techniques for implementing concurrent objects typically rely on critical sections: ensuring that only one process at a time can operate on the object. Nevertheless, critical sections are poorly suited for asynchronous systems: if one process is halted or delayed in a critical section, other, nonfaulty processes will be unable to progress. By contrast, a concurrent object implementation is lock free if it always guarantees that some process will complete an operation in a finite number of steps, and it is wait free if it guarantees that each process will complete an operation in a finite number of steps. This paper proposes a new methodology for constructing lock-free and wait-free implementations of concurrent objects. The object's representation and operations are written as stylized sequential programs, with no explicit synchronization. Each sequential operation is automatically transformed into a lock-free or wait-free operation using novel synchronization and memory management algorithms. These algorithms are presented for a multiple instruction/multiple data (MIMD) architecture in which $n$ processes communicate by applying atomic read, write, load_linked, and store_conditional operations to a shared memory.
\end{abstract}

Categories and Subject Descriptors: D.2.1 [Software Engineering]: Requirements/specifications-methodologies; D.3.3 [Programming Languages]: Language Constructs and Features-concurrent programming structures; D.4.1 [Operating Systems]: Process Management-concurrency; deadlocks; Synchronizing

General Terms: Algorithms, Management, Performance, Theory

\section{INTRODUCTION}

A concurrent object is a data structure shared by concurrent processes. Conventional techniques for implementing concurrent objects typically rely on critical sections to ensure that only one process at a time is allowed to access to the object. Nevertheless, critical sections are poorly suited for asynchronous systems; if one process is halted or delayed in a critical section, other, faster processes will be unable to progress. Possible sources of unexpected delay include page faults, cache misses, scheduling preemption, and perhaps even processor failure.

By contrast, a concurrent object implementation is lock free if some process must complete an operation after the system as a whole takes a finite number

Author's address: Digital Equipment Corporation, Cambridge Research Laboratory, One Kendall Square, Cambridge, MA 02139; email: herlihy (âcrl.dec.com.

Permission to copy without fee all or part of this material is granted provided that the copies are not made or distributed for direct commercial advantage, the ACM copyright notice and the title of the publication and its date appear, and notice is given that copying is by permission of the Association for Computing Machinery. To copy otherwise, or to republish, requires a fee and/or specific permission.

(c) $1993 \mathrm{ACM} 0164-0925 / 93 / 1100-0745 \$ 03.50$ 
of steps, ${ }^{1}$ and it is wait free if each process must complete an operation after taking a finite number of steps. The lock-free condition guarantees that some process will always make progress despite arbitrary halting failures or delays by other processes, while the wait-free condition guarantees that all nonhalted processes make progress. Either condition rules out the use of critical sections, since a process that halts in a critical section can force other processes trying to enter that critical section to run forever without making progress. The lock-free condition is appropriate for systems where starvation is unlikely, while the (strictly stronger) wait-free condition may be appropriate when some processes are inherently slower than others, as in certain heterogeneous architectures.

The theoretical issues surrounding lock-free synchronization protocols have received a fair amount of attention, but the practical issues have not. In this paper, we make a first step toward addressing these practical aspects by proposing a new methodology for constructing lock-free and wait-free implementations of concurrent objects. Our approach focuses on two distinct issues: ease of reasoning and performance.

-It is no secret that reasoning about concurrent programs is difficult. A practical methodology should permit a programmer to design, say, a correct lock-free priority queue, without ending up with a publishable result.

- The lock-free and wait-free properties, like most kinds of fault-tolerance, incur a cost, especially in the absence of failures or delays. A methodology can be considered practical only if (1) we understand the inherent costs of the resulting programs, (2) this cost can be kept to acceptable levels, and (3) the programmer has some ability to influence these costs.

We address the reasoning issue by having programmers implement data objects as stylized sequential programs, with no explicit synchronization. Each sequential implementation is automatically transformed into a lockfree or wait-free implementation via a collection of novel synchronization and memory management techniques introduced in this paper. If the sequential implementation is a correct sequential program, and if it follows certain simple conventions described below, then the transformed program will be a correct concurrent implementation. The advantage of starting with sequential programs is clear: the formidable problem of reasoning about concurrent programs and data structures is reduced to the more familiar sequential domain. (Because programmers are required to follow certain conventions, this methodology is not intended to parallelize arbitrary sequential programs after the fact.)

To address the performance issue, we built and tested prototype implementations of several concurrent objects on a multiprocessor. We show that a naive implementation of our methodology performs poorly because of excessive memory contention, but simple techniques from the literature (such as exponential backoff) have a dramatic effect on performance. We also compare

\footnotetext{
${ }^{1}$ The lock-free condition is sometimes called nonblocking. 
our implementations with more conventional implementations based on spin locks. Even in the absence of timing anomalies, our example implementations sometimes outperform conventional spin-lock techniques, and lie within a factor of two of more sophisticated spin-lock techniques.

We focus on a multiple instruction/multiple data (MIMD) architecture in which $n$ asynchronous processes communicate by applying atomic read, write, load_linked, and store_conditional operations to a shared memory. The load_linked operation copies the value of a shared variable to a local variable. A subsequent store_conditional to the shared variable will change its value only if no other process has modified that variable in the interim. Either way, the store_conditional returns an indication of success or failure. (Note that a store_conditional is permitted to fail even if the variable has not changed. We assume that such spurious failures are rare, though possible.)

We chose to focus on the load_linked and store_conditional synchronization primitives for three reasons. First, they can be implemented efficiently in a cache-coherent architectures [Jenson et al. 1987; Kane 1989; Sites 1992], since store_conditional need only check whether the cached copy of the shared variable has been invalidated. Second, many other "classical" synchronization primitives are provably inadequate-we have shown elsewhere [Herlihy 1991] that it is impossible ${ }^{2}$ to construct lock-free or wait-free implementations of many simple and useful data types using any combination of read, write, test \& set, fetch \& add [Gottlieb et al. 1984], and memoryto-register swap. The load_linked and store_conditional operations, however, are universal-at least in principle, they are powerful enough to transform a sequential implementation of any object into a lock-free or wait-free implementation. Finally, we have found load_linked and store_conditional easy to use. Elsewhere [Herlihy 1990], we present a collection of synchronization and memory management algorithms based on compare \& swap [IBM]. Although these algorithms have the same functionality as those given here, they are less efficient, and conceptually more complex.

In our prototype implementations, we used the $\mathrm{C}$ language [Kernighan and Ritchie 1988] on an Encore Multimax [Encore 1989] with eighteen NS32532 processors. This architecture does not provide load_linked or store_conditional primitives, so we simulated them using short critical sections. Naturally, our simulation is less efficient than direct hardware support. For example, a successful store_conditional requires twelve machine instructions rather than one. Nevertheless, these prototype implementations are instructive because they allow us to compare the relative efficiency of different implementations using load_linked and store_conditional, and because they still permit an approximate comparison of the relative efficiency of waiting versus nonwaiting techniques. We assume readers have some knowledge of the syntax and semantics of C.

In Section 2, we give a brief survey of related work. Section 3 describes our model. In Section 4, we present protocols for transforming sequential imple-

\footnotetext{
${ }^{2}$ Although our impossibility results were presented in terms of wait-free implementations, they hold for lock-free implementations as well.
} 
mentations of small objects into lock-free and wait-free implementations, together with experimental results showing that our techniques can be made to perform well even when each process has a dedicated processor. In Section 5 , we extend this methodology to encompass large objects. Section 6 summarizes our results, and concludes with a discussion.

\section{RELATED WORK}

Early work on lock-free protocols focused on impossibility results [Chor et al. 1987; Dolev et al. 1987; Dwork et al. 1986; 1988; Fischer et al. 1985; Herlihy 1991], showing that certain problems cannot be solved in asynchronous systems using certain primitives. By contrast, a synchronization primitive is universal if it can be used to transform any sequential object implementation into a wait-free concurrent implementation. The author [Herlihy 1991] gives a necessary and sufficient condition for universality: a synchronization primitive is universal in an $n$-process system if and only if it solves the well-known consensus problem [Fischer et al. 1985] for $n$ processes. Although this result established that wait-free (and lock-free) implementations are possible in principle, the construction given was too inefficient to be practical. Plotkin [1989] gives a detailed universal construction for a sticky-bit primitive. This construction is also of theoretical rather than practical interest. Elsewhere [Herlihy 1990], the author gives a simple and relatively efficient technique for transforming stylized sequential object implementations into lock-free and wait-free implementations using the compare \& swap synchronization primitive. Although the overall approach is similar to the one presented here, the details are quite different. In particular, the constructions presented in this paper are simpler and more efficient, for reasons discussed below.

Many researchers have studied the problem of constructing wait-free atomic registers from simpler primitives [Burns and Peterson 1987; Lamport 1986; $\mathrm{Li}$ et al. 1991; Peterson 1983; Peterson and Burns 1986]. Atomic registers, however, have few if any interesting applications for concurrent data structures, since they cannot be combined to construct lock-free or wait-free implementations of most common data types [Herlihy 1991]. There exists an extensive literature on concurrent data structures constructed from more powerful primitives. Gottlieb et al. [1983] give a highly concurrent queue implementation based on the replace-add operation, a variant of fetch $\&$ add. This implementation permits concurrent enqueuing and dequeuing processes, but it is blocking, since it uses critical sections to synchronize access to individual queue elements. Lamport [1983] gives a wait-free queue implementation that permits one enqueuing process to execute concurrently with one dequeuing process. Herlihy and Wing [1987] give a lock-free queue implementation, employing fetch $\&$ add and swap, that permits an arbitrary number of enqueuing and dequeuing processes. Lanin and Shasha [1988] give a lock-free set implementation that uses operations similar to compare \& swap. There exists an extensive literature on locking algorithms for concurrent B-trees [Bayer and Schkolnick 1977; Lehman and Yao 1981] and for related search structures [Biswas and Browne 1987; Ellis 1980; Ford and 
Calhoun 1984; Guibas and Sedgewick 1978; Jones 1989]. More recent approaches to implementing lock-free data structures include Allemany and Felton's work on operating system support [Allemany and Felton 1992], and Herlihy and Moss's work on hardware support [Herlihy and Moss 1993].

The load_linked and store_conditional synchronization primitives were first proposed as part of the S-1 project [Jensen et al. 1987] at Lawrence Livermore Laboratories, and they are currently supported in the MIPS-II architecture [Kane 1989] and Digital's Alpha [Sites 1992]. They are closely related to the compare \& swap operation first introduced by the IBM 370 architecture [IBM].

Our techniques are distantly related to optimistic concurrency control methods from the database literature [Kung and Robinson 1981]. In these schemes, transactions execute without synchronization, but each transaction must be validated before it is allowed to commit to ensure that synchronization conflicts did not occur. Our method also checks after-the-fact whether synchronization conflicts occurred, but the technical details are entirely different.

\section{OVERVIEW}

A concurrent system consists of a collection of $n$ sequential processes that communicate through shared typed objects. Processes are sequential-each process applies a sequence of operations to objects, alternately issuing an invocation and then receiving the associated response. We make no fairness assumptions about processes. A process can halt, or display arbitrary variations in speed. In particular, one process cannot tell whether another has halted or is just running very slowly.

Objects are data structures in memory. Each object has a type, which defines a set of possible values and a set of primitive operations that provide the only means to manipulate that object. Each object has a sequential specification that defines how the object behaves when its operations are invoked one at a time by a single process. For example, the behavior of a queue object can be specified by requiring that enqueue insert an item in the queue, and that dequeue remove the oldest item present in the queue. In a concurrent system, however, an object's operations can be invoked by concurrent processes, and it is necessary to give a meaning to interleaved operation executions.

An object is linearizable [Herlihy and Wing 1987] if each operation appears to take effect instantaneously at some point between the operation's invocation and response. Linearizability implies that processes appear to be interleaved at the granularity of complete operations, and that the order of nonoverlapping operations is preserved. As discussed in more detail elsewhere [Herlihy and Wing 1987], the notion of linearizability generalizes and unifies a number of ad hoc correctness conditions in the literature, and it is related to (but not identical with) correctness criteria such as sequential consistency [Lamport 1979] and strict serializability [Papadimitriou 1979]. 
We use linearizability as the basic correctness condition for the concurrent objects constructed in this paper.

Our methodology is the following:

(1) The programmer provides a sequential implementation of the object, choosing a representation and implementing the operations. This program is written in a conventional sequential language, subject to certain restrictions given below. This implementation performs no explicit synchronization.

(2) Using the synchronization and memory management algorithms described in this paper, this sequential implementation is transformed into a lock-free (or wait-free) concurrent implementation. Although we do not address the issue here, this transformation is simple enough to be performed by a compiler or preprocessor.

We refer to data structures and operations implemented by the programmer as sequential objects and sequential operations, and we refer to transformed data structures and operations as concurrent objects and concurrent operations. By convention, names of sequential data types and operations are in lowercase, while names of concurrent types and operations are capitalized. (Compile-time constants typically appear in uppercase.)

\section{SMALL OBJECTS}

A small object is one that is small enough to be copied efficiently. In this section we discuss how to construct lock-free and wait-free implementations of small objects. In a later section, we present a slightly different methodology for large objects, which are too big to be copied all at once.

A sequential object is a data structure that occupies a fixed-size contiguous region of memory called a block. Each sequential operation is a stylized sequential program subject to the following simple constraints.

-A sequential operation may not have any side-effects other than modifying the block occupied by the object.

- A sequential operation must be total, meaning that it is well-defined for every legal state of the object. (For example, the dequeue operation may return an error code or signal an exception when applied to an empty queue, but it may not provoke a core dump.)

The motivation for these restrictions will become clear when we discuss how sequential operations are transformed into concurrent operations.

Throughout this paper, we use the following extended example. A priority queue (pqueue_type) is a set of items taken from a totally-ordered domain (our examples use integers). It provides two operations: enqueue (pqueue_enq) inserts an item into the queue, and dequeue (pqueue_deq) removes and returns the least item in the queue. A well-known technique for implementing a priority queue is to use a heap, a binary tree in which each node has a 


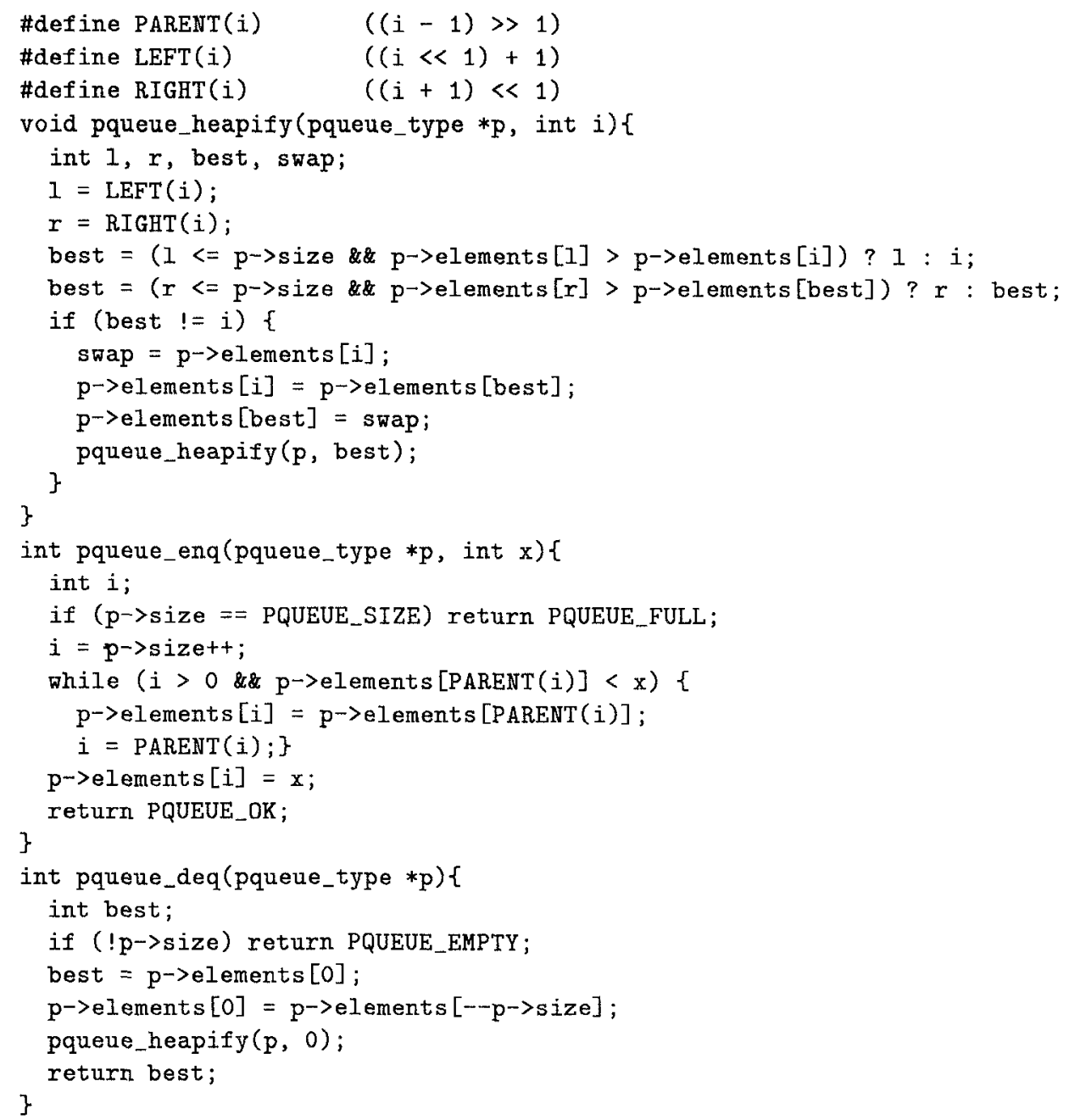

Fig. 1. A sequential priority queue implementation.

higher priority than its children. Figure 1 shows a sequential implementation of a priority queue that satisfies our conditions. ${ }^{3}$

\subsection{The Lock-Free Transformation}

We first discuss how to transform a sequential object into a lock-free concurrent object. We start with a protocol that guarantees correctness, and later we extend it to enhance performance. For now, we assume that processes share only one object, a restriction we will relax later.

Omitting certain important details, the basic technique is the following. The objects share a variable that holds a pointer to the object's current

\footnotetext{
${ }^{3}$ This code is adapted from Cormen et al. [1990].
} 
version. Each process (1) reads the pointer using load_linked, (2) copies the indicated version into another block, (3) applies the sequential operation to the copy, and (4) calls store_conditional to swing the pointer from the old version to the new. If the last step fails, the process restarts at Step 1. Each execution of these four steps is called an attempt. Linearizability is straightforward, since the order in which operations appear to happen is the order of their final calls to store_conditional. Barring spurious failures of the store conditional primitive, this protocol is lock-free because at least one out of every $n$ attempts must succeed.

Memory management for small objects is almost trivial. Each process owns a single block of unused memory. In Step 2, the process copies the object's current version into its own block. When it succeeds in swinging the pointer from the old version to the new, it gives up ownership of the new version's block, and acquires ownership of the old version's block. Since the process that replaces a particular version is uniquely determined, each block has a unique and well-defined owner at all times.

A slow process may observe the object in an inconsistent state. For example, processes $P$ and $Q$ may read a pointer to a block $b, Q$ may swing the pointer to block $b^{\prime}$ and then start a new operation. If $P$ copies $b$ while $Q$ is copying $b^{\prime}$ back to $b$, then $P^{\prime}$ 's copy may not be a valid state of the sequential object. This race condition raises an important software engineering issue. Although P's subsequent store_conditional is certain to fail, it may be difficult to ensure that the sequential operation does not store into an out-of-range location, divide by zero, or perform some other illegal action. It would be imprudent to require programmers to write sequential operations that avoid such actions when presented with arbitrary bit strings. Instead, we insert a consistency check after copying the old version, but before applying the sequential operation. Consistency can be checked either by hardware or by software. A simple hardware solution is to include a validate instruction that checks whether a variable read by a load_linked instruction has been modified. Implementing such a primitive in an architecture that already supports store_conditional should be straightforward, since they have similar functionalities. In our examples, however, we use a software solution. Each version has two associated counters, check [0] and check [1]. If the counters are equal, the version is consistent. To modify a version, a process increments check [0], makes the modifications, and then increments check [1]. When copying, a process reads check [1], copies the version, and then reads check [0]. Incrementing the counters in one order and reading them in the other ensures that if the counters match, then the copy is consistent. ${ }^{*}$

We are now ready to review the protocol in more detail (Figure 2). A concurrent object is a shared variable that holds a pointer to a structure with two fields. (1) version is a sequential object, and (2) check is a two-element

\footnotetext{
${ }^{4}$ Counters are bounded, so there is a remote chance that a consistency check will succeed incorrectly if a counter cycles all the way around during a single attempt. As a practical matter, this problem is avoided simply by using a large enough (e.g., 32-bit) counter.
}

ACM Transactions on Programming Languages and Systems, Vol 15, No. 5, November 1993 


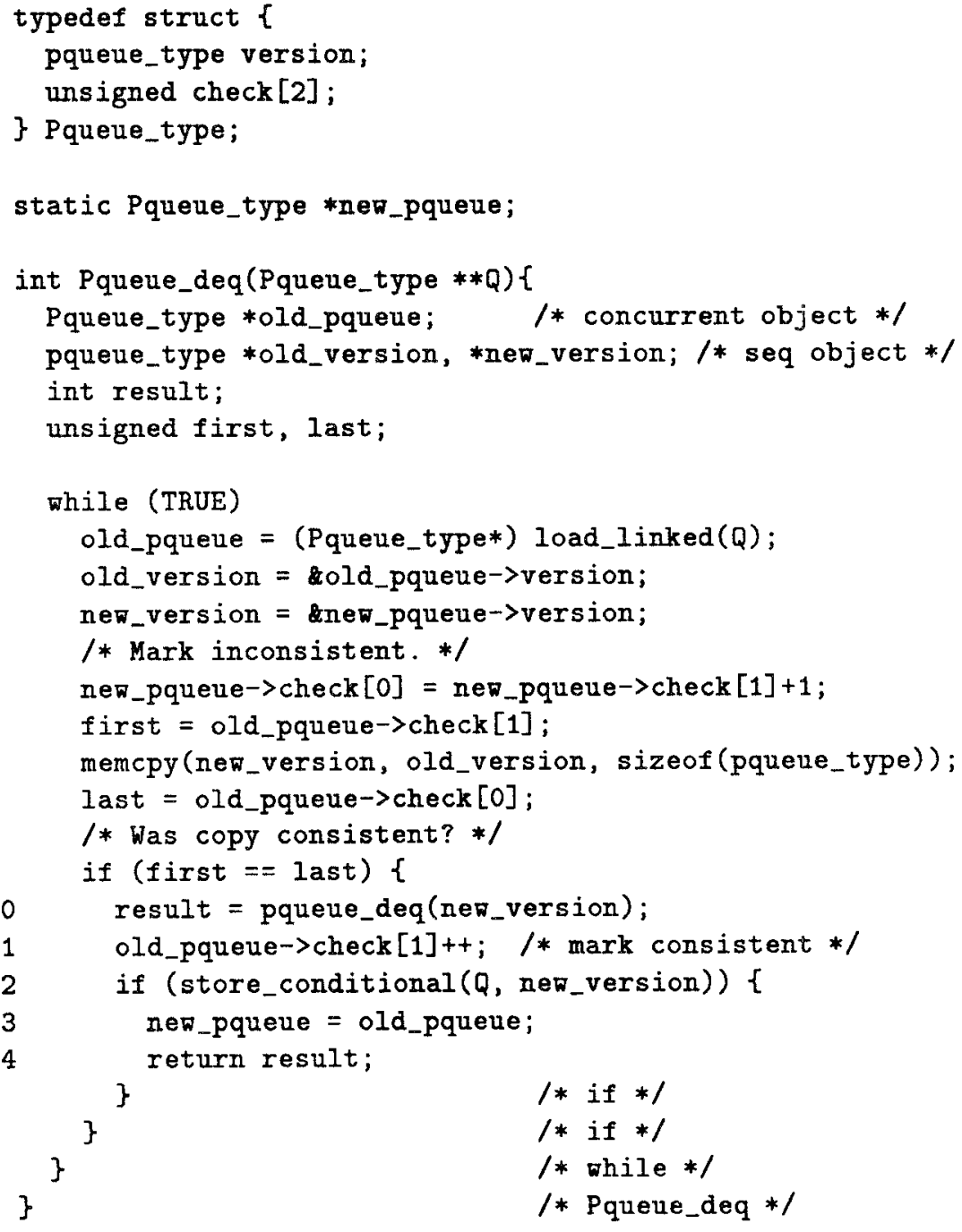

Fig. 2. Simple lock-free protocol.

array of unsigned (large) integers. Each process keeps a pointer (new_pqueue) that points to the block it owns. The process enters a loop (Line 1). It reads the pointer using load_linked (Line 2), and marks the new version as inconsistent by setting check [0] to check [1] +1 (Line 5). It then reads the old version's check [1] field (Line 6), copies the version field (Line 7), and then reads the check [0] field (Line 8). If the two counters match (Line 9), then the copy is consistent, and the process applies the sequential operation to the version field (Line 10), and then increments check [1] (Line 11), indicating that the version is consistent. It then attempts to reset the pointer using store_conditional (Line 12). If it succeeds, then it sets new_pqueue to 
point to the old version (Line 13) and returns (Line 14). Otherwise, it resumes the loop.

\subsection{Remarks}

In architectures such as Digital's Alpha [Sites 1992], load_linked is implemented by loading a location into a processor's cache, and store_conditional simply checks whether that cache line has been invalidated. If not, the store_conditional succeeds, otherwise it fails. A problem here is that a cache line can be invalidated for a number of reasons, including timer interrupts, page faults, and cache overflow. The longer the "window" between a load_linked and the corresponding store_conditional, the greater the likelihood of spurious failure. This window can be narrowed significantly by the following simple change. After a process has finished updating the new version, it rereads the pointer (using load_linked) and check [0] (Line 16 of Figure 7). If either has changed, it abandons the operation, but otherwise it applies the store_conditional as before. This refinement narrows the "window of vulnerability" during which an interrupt or cache collision will cause the operation to fail. Notice that only the very last pointer read requires a load_linked.

The protocol does not work if a single block can hold objects of different type. ${ }^{5}$ Consider the following execution: $P$ and $Q$ each reads a pointer to a block $b$ holding an object of type $T, Q$ completes its operation, replacing $b$ and acquiring ownership. $Q$ then reinitializes $b$ to hold an object of type $S$. Now $P$ reads check [1], copies the version, and reads check [0]. Because the counters match, $P$ (correctly) decides that the version is consistent, but (incorrectly) applies an operation of type $T$ to an object of type $S$. This problem can be fixed by having the process reread the pointer after copying the block, but before reading check [0] (Line 11 in Figure 7). If the pointer has changed, the process abandons the operation.

This protocol also does not work if compare \& swap replaces store conditional. Consider the following execution: $P$ and $Q$ each reads a pointer to a block $b, Q$ completes its operation, replacing $b$ with $b^{\prime}$ and acquiring ownership of $b . Q$ then completes a second operation, replacing $b^{\prime}$ with $b$. If $P$ now does a compare $\&$ swap, then it will erroneously install an out-ofsequence version. Elsewhere [Herlihy 1990], we describe a more complex protocol in which $P$ "freezes" a block before reading it, ensuring that the block will not be recycled while the attempt is in progress. As mentioned above, the resulting protocols are more complex and less efficient than the ones described here for store_conditional.

Several additional optimizations are possible. If the hardware provides a validate operation, then read-only operations can complete with a successful validate instead of a store conditional. An object may be significantly smaller than a full block. If programmers follow a convention where the object's true size is kept in a fixed location within the block, then the

\footnotetext{
${ }^{5}$ I am grateful to Abdelsalam Heddaya and Himanshu Sinha for this observation ACM Transactions on Programming Languages and Systems, Vol 15, No. 5, November 1993
} 


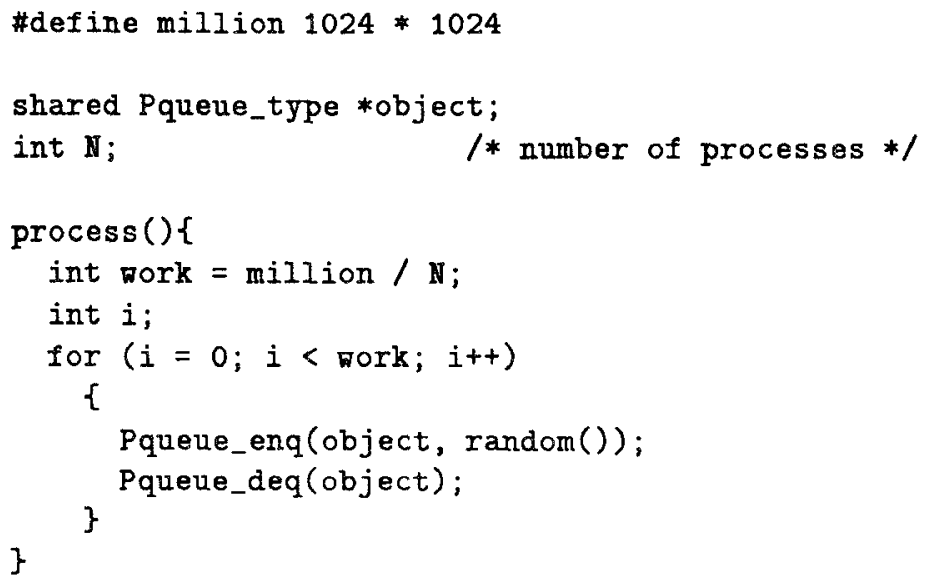

Fig. 3. Concurrent heap benchmark.

concurrent operation can avoid unnecessary copying. (Our prototypes make use of this optimization.)

\subsection{Experimental Results}

The lock-free property is best thought of as a kind of fault-tolerance. In return for extra work (updating a copy instead of updating in place), the program acquires the ability to withstand certain failures (unexpected process failure or delay). In this section, we present experimental results that provide a rough measure of this additional overhead, and that allow us to identify and evaluate certain additional techniques that substantially enhance performance. We will show that a naive implementation of the lock-free transformation performs poorly, even allowing for the cost of simulated load_linked and store_conditional, but that adding a simple exponential backoff dramatically increases throughput.

As described above, we constructed a prototype implementation of a small priority queue on an Encore Multimax, in C, using simulated load_linked and store_conditional primitives. As a benchmark, we measure the elapsed time needed for $n$ processes to enqueue and then dequeue $2^{20} / n$ items from a shared 16-element priority queue (Figure 3), where $n$ ranges from 1 to 16 . As a control, we also ran the same benchmark using the same heap implementation of the priority queue, except that updates were done in place, using an in-line compiled test-and-test-and-set ${ }^{6}$ spin lock to achieve mutual exclusion. The test-and-test-and-set spin lock is a built-in feature of Encore's $\mathrm{C}$ compiler, and it represents how most current systems synchronize access to shared data structures.

When evaluating the performance of these benchmarks, it is important to understand that they were run under circumstances where timing anomalies

${ }^{6}$ A test-and-test-and-set [Rudolph and Segall 1983] loop repeatedly reads the lock until it observes the lock is free, and then tries the test $\boldsymbol{\&}$ set operation. 


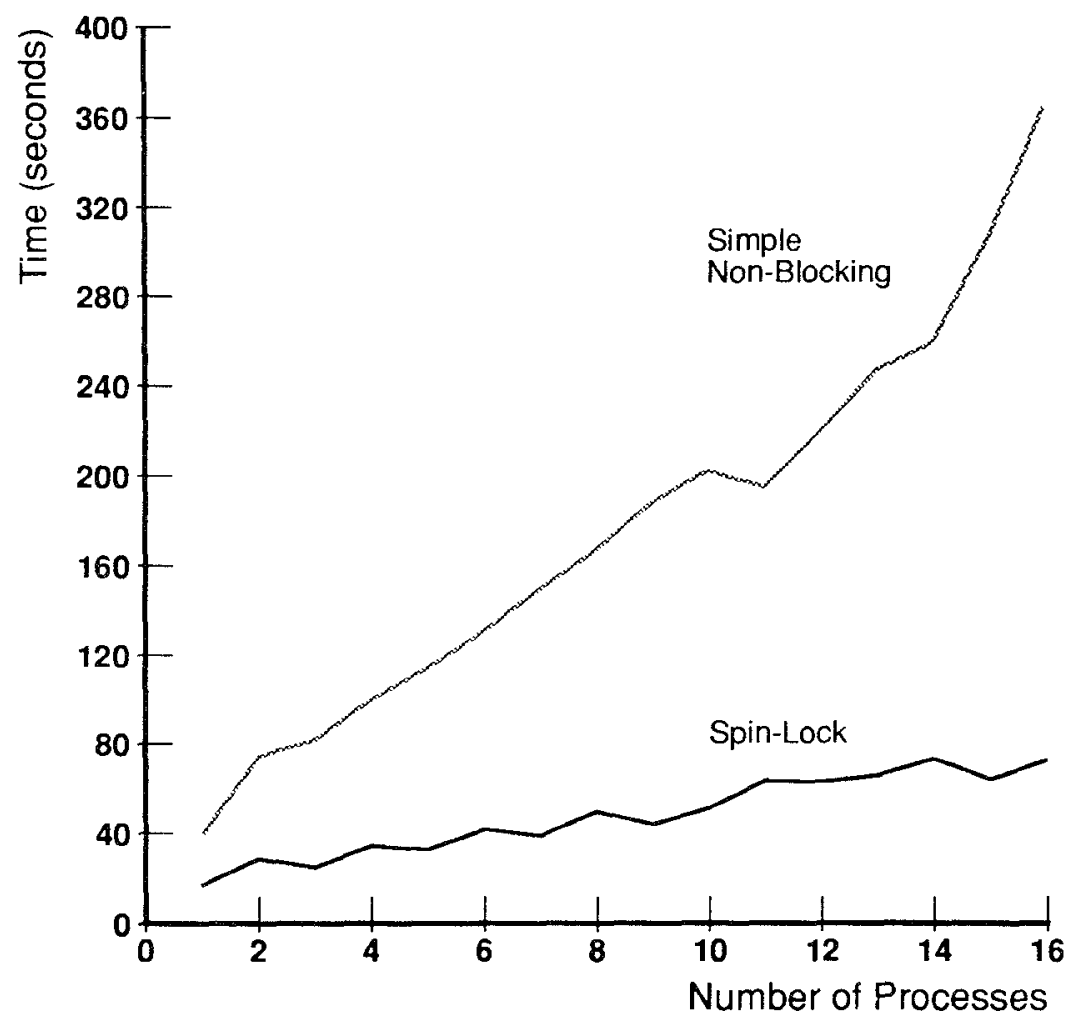

Fig. 4. Simple lock-free versus spın-lock.

and delays almost never occur. Each process ran on its own dedicated processor, and the machine was otherwise idle, ensuring that processes were likely to run uninterruptedly. The processes repeatedly accessed a small region of memory, making page faults unlikely. Under these circumstances, the costs of avoiding waiting are visible, although the benefits are not. Nevertheless, we chose these circumstances because they best highlight the inherent costs of our proposal.

In Figure 4, the horizontal axis represents the number of concurrent processes executing the benchmark, and the vertical axis represents the time taken (in seconds). The top curve is the time taken using the lock-free protocol, and the lower curve is the time taken by the spin lock. When reading this graph, it is important to bear in mind that each point represents approximately the same amount of work-enqueuing and dequeuing $2^{20}$ (about a million) randomly-generated numbers. In the absence of memory contention, both curves would be nearly flat. ${ }^{7}$

${ }^{7}$ Concurrent executions are slightly less efficient because the heap's maximum possible size is a function of the level of concurrency.

ACM Transactions on Programming Languages and Systems, Vol. 15, No. 5, November 1993 


\begin{tabular}{|l||l|l|l|l|l|l|l|l|}
\hline & 2 & 4 & 6 & 8 & 10 & 12 & 14 & 16 \\
\hline \hline Dequeue Average & 1.04 & 2.15 & 2.86 & 3.29 & 3.70 & 4.01 & 4.44 & 5.23 \\
\hline Enq Average & 2.89 & 4.75 & 4.79 & 4.84 & 5.00 & 5.19 & 5.50 & 5.93 \\
\hline \hline Deq Maximum & 5 & 124 & 73 & 83 & 83 & 150 & 98 & 73 \\
\hline Enq Maximum & 2046 & 3090 & 1596 & 2789 & 5207 & 4881 & 2592 & 178 \\
\hline
\end{tabular}

Fig. 5. Simple lock-free protocol: Number of attempts.

\begin{tabular}{|l||l|l|l|l|l|l|l|l|}
\hline & 2 & 4 & 6 & 8 & 10 & 12 & 14 & 16 \\
\hline \hline Dequeue Average & 1.00 & 1.00 & 1.00 & 1.00 & 1.00 & 1.00 & 1.00 & 1.00 \\
\hline Enq Average & 1.00 & 1.00 & 1.00 & 1.00 & 1.00 & 1.00 & 1.00 & 1.00 \\
\hline \hline Deq Maximum & 1 & 30 & 60 & 80 & 60 & 27 & 48 & 58 \\
\hline Enq Maximum & 1 & 122 & 138 & 159 & 216 & 244 & 254 & 282 \\
\hline
\end{tabular}

Fig. 6. Lock-free with backoff: Number of attempts.

The simple lock-free protocol performs much worse than the spin-lock protocol, even allowing for the inherent inefficiency of the simulated load_linked and store_conditional primitives. The poor performance of the lock-free protocol is primarily a result of memory contention. In each protocol, only one of the $n$ processes is making progress at any given time. In the spin-lock protocol, it is the process in the critical section, while in the lock-free protocol, it is the process whose store_conditional will eventually succeed. In the spin-lock protocol, however, the process outside the critical section are spinning on cached copies of the lock, and are therefore not generating any bus traffic. In the lock-free protocol, by contrast, all processes are generating bus traffic, so only a fraction of the bus bandwidth is dedicated to useful work.

The simple lock-free protocol has a second weakness: starvation. The enqueue operation is about $10 \%$ slower than the dequeue operation. If we look at the average number of attempts associated with each process (Figure 5), we can see that enqueues make slightly more unsuccessful attempts than dequeues, but that each makes an average of fewer than six attempts. If we look at the maximum number of attempts (Figure 6), however, a dramatically different story emerges. The maximum number of unsuccessful dequeue attempts is in the high thousands, while the maximum number of enqueue hovers around one hundred. This table shows that starvation is indeed a problem, since a longer operation may have difficulty completing if it competes with shorter operations.

These performance problems have a simple solution. We introduce an exponential backoff [Anderson 1990; Mellor-Crummey and Scott 1990; Metcalf and Boggs 1976] between successive attempts (Figure 7). Each process keeps a dynamically-adjusted maximum delay. When an operation starts, it halves its current maximum delay (Line 1). Each time an attempt 


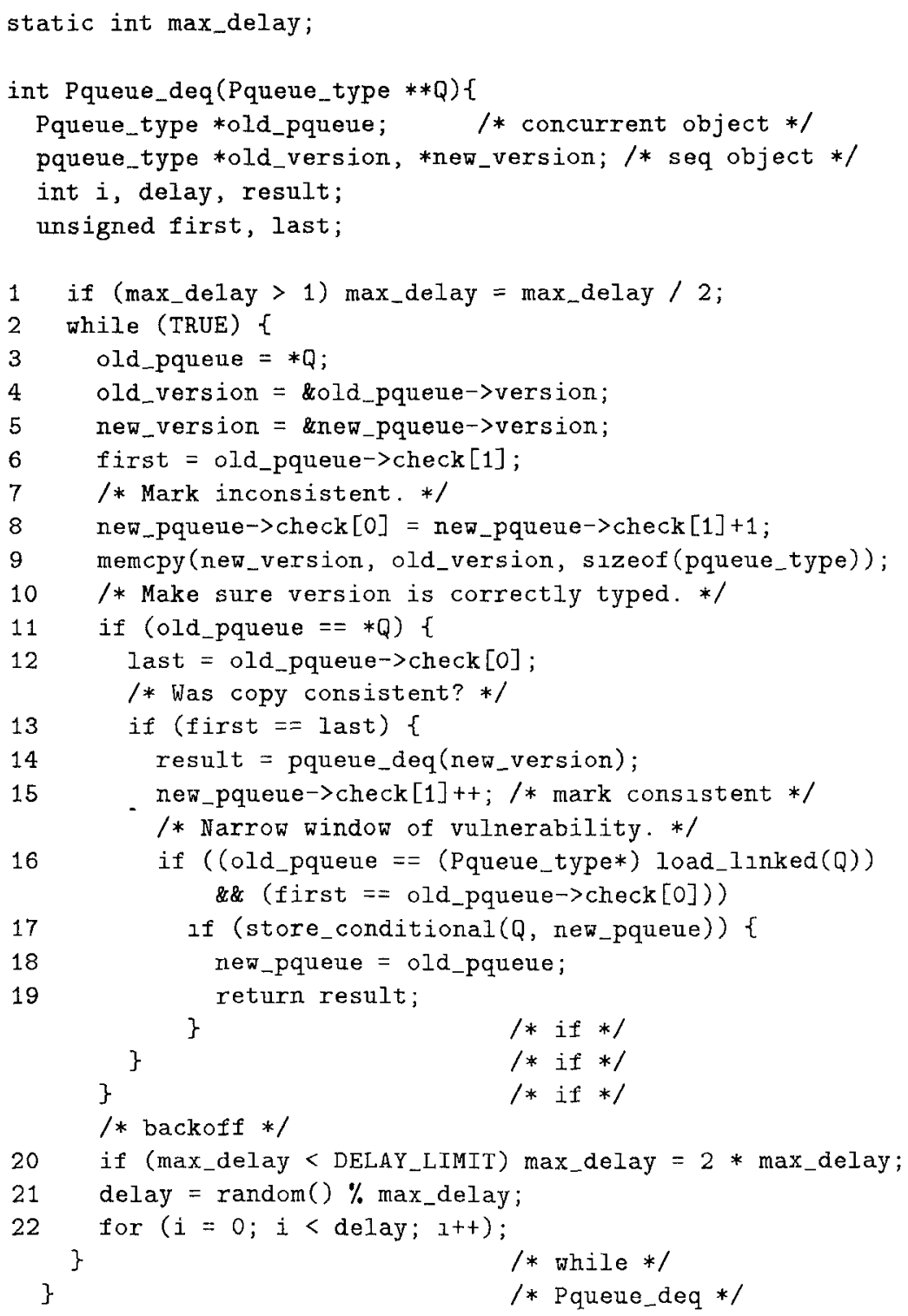

Fig. 7. Lock-free protocol with backoff and optimizations.

fails, the process doubles the maximum delay, up to a fixed limit (Line 20), and waits for a random duration less than the maximum delay (Lines 21 and $22)^{8}$

Exponential backoff has a striking effect on performance. As illustrated in Figure 8, the throughput of the lock-free protocol soon overtakes that of

${ }^{8}$ For speed, each process in our prototype uses a precomputed table of random numbers, and certain arithmetic operations are performed by equivalent bit-wise logical operations 


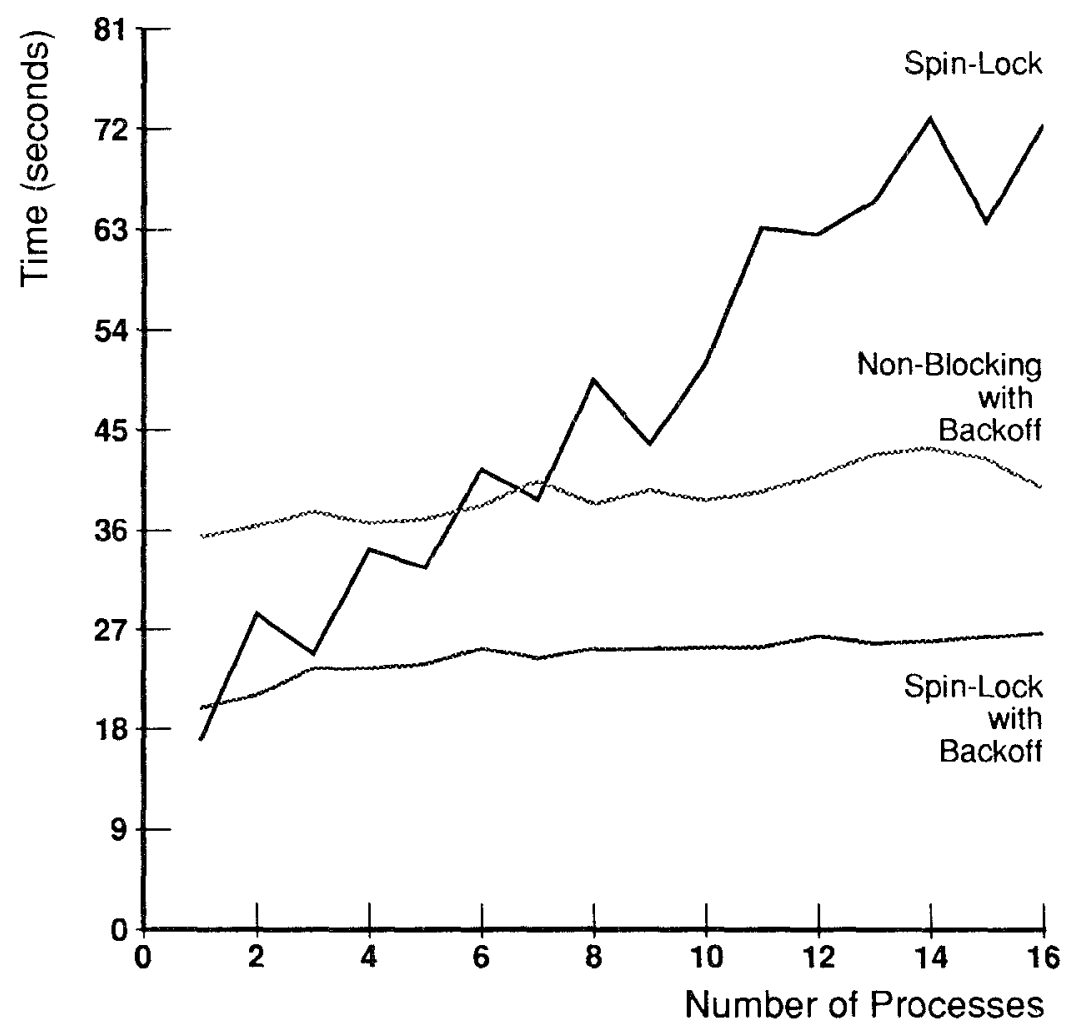

Fig. 8. The effect of exponential backoff.

the standard spin lock implementation. Moreover, starvation is no longer a threat. In the typical execution shown in Figure 6, the average number of attempts is 1.00 (out of $2^{20}$ operations), and the maximum for enqueues is reduced by an order of magnitude.

It is well known that spin-locks also benefit from exponential backoff [Anderson 1990; Mellor-Crummey and Scott 1990]. We replaced the in-line compiled test-and-test-and-set spin lock with a hand-coded spin lock that itself employs exponential backoff. Not surprisingly, this protocol has the best throughput of all when run with dedicated processors, almost twice that of the lock-free protocol.

In summary, using exponential backoff, the lock-free protocol significantly outperforms a straightforward spin-lock protocol (the default provided by the Encore C compiler), and lies within a factor of two of a sophisticated spin-lock implementation.

\subsection{A Wait-Free Protocol}

This protocol can be made wait-free by a technique we call operation combining. When a process starts an operation, it records the call in an invocation structure (inv_type) whose fields include the operation name (op_name), 


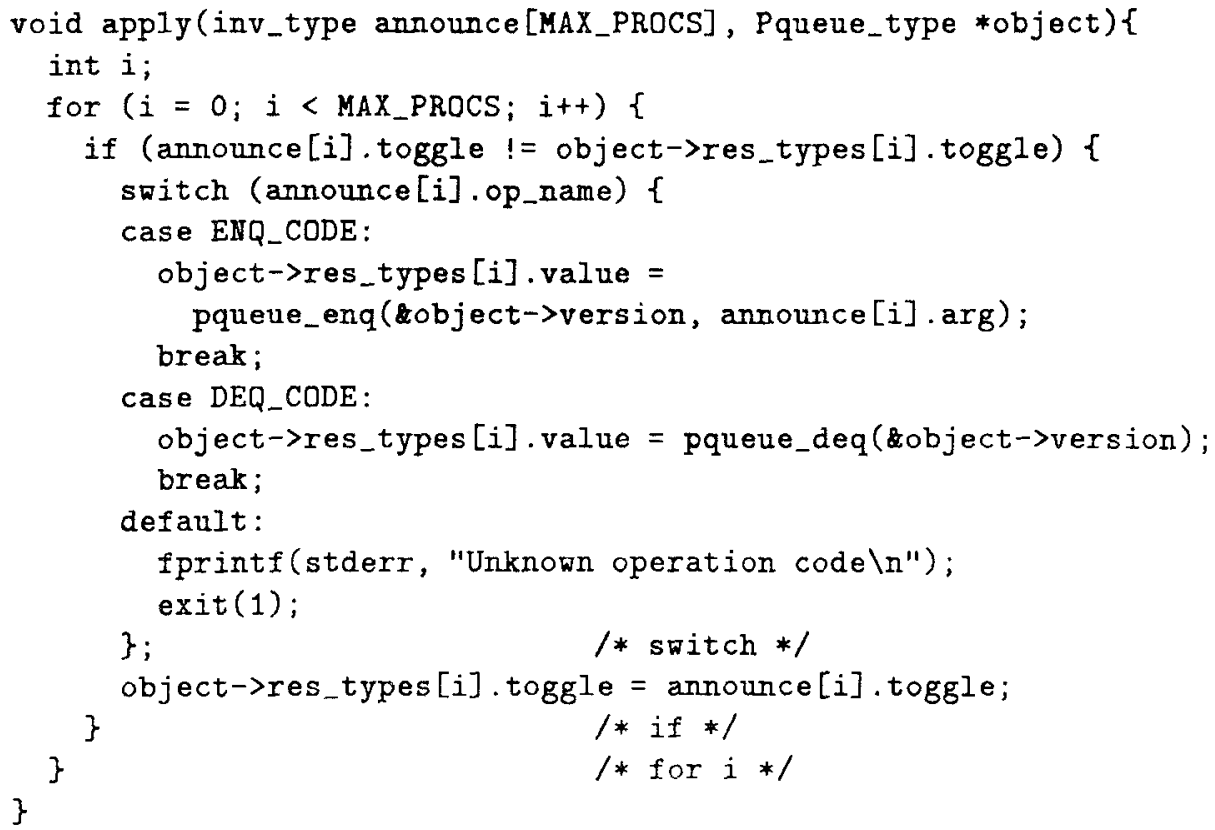

Fig. 9. The apply operations.

argument value (arg), ${ }^{9}$ and a toggle bit (toggle) used to distinguish old and new invocations. When it completes an operation, it records the result in a response (res_type) structure, whose fields include the result (value) and toggle bit. Each concurrent object has an additional field: responses is an $n$-element array of responses, whose $p$ th element is the result of $P$ 's last completed operation. The processes share an $n$-element array announce of invocations. When $P$ starts an operation, it records the operation name and argument in announce [P]. Each time a process records a new invocation, it complements the invocation's toggle bit.

A wait-free dequeue operation appears in Figure 10. (For brevity, we have omitted the optimizations discussed in Section 4.2.) After performing the consistency check (Line 12), the apply procedure (Figure 9) scans the responses and announce arrays, comparing the toggle fields of corresponding invocations and responses. If the bits disagree, then it applies that invocation to the new version, records the result in the matching position in the responses array, and complements the response's toggle bit. After calling the apply procedure to apply the pending operations to the new version, the process calls store_conditional to replace the old version, just as before. To determine when its own operation is complete, $P$ compares the toggle bits of its invocation with the object's matching response. It performs this comparison twice, rereading the pointer between the first and second comparison. If

\footnotetext{
${ }^{9}$ The argument value could be a pointer to a vector of arguments.

ACM Transactions on Programming Languages and Systems, Vol 15, No. 5, November 1993
} 


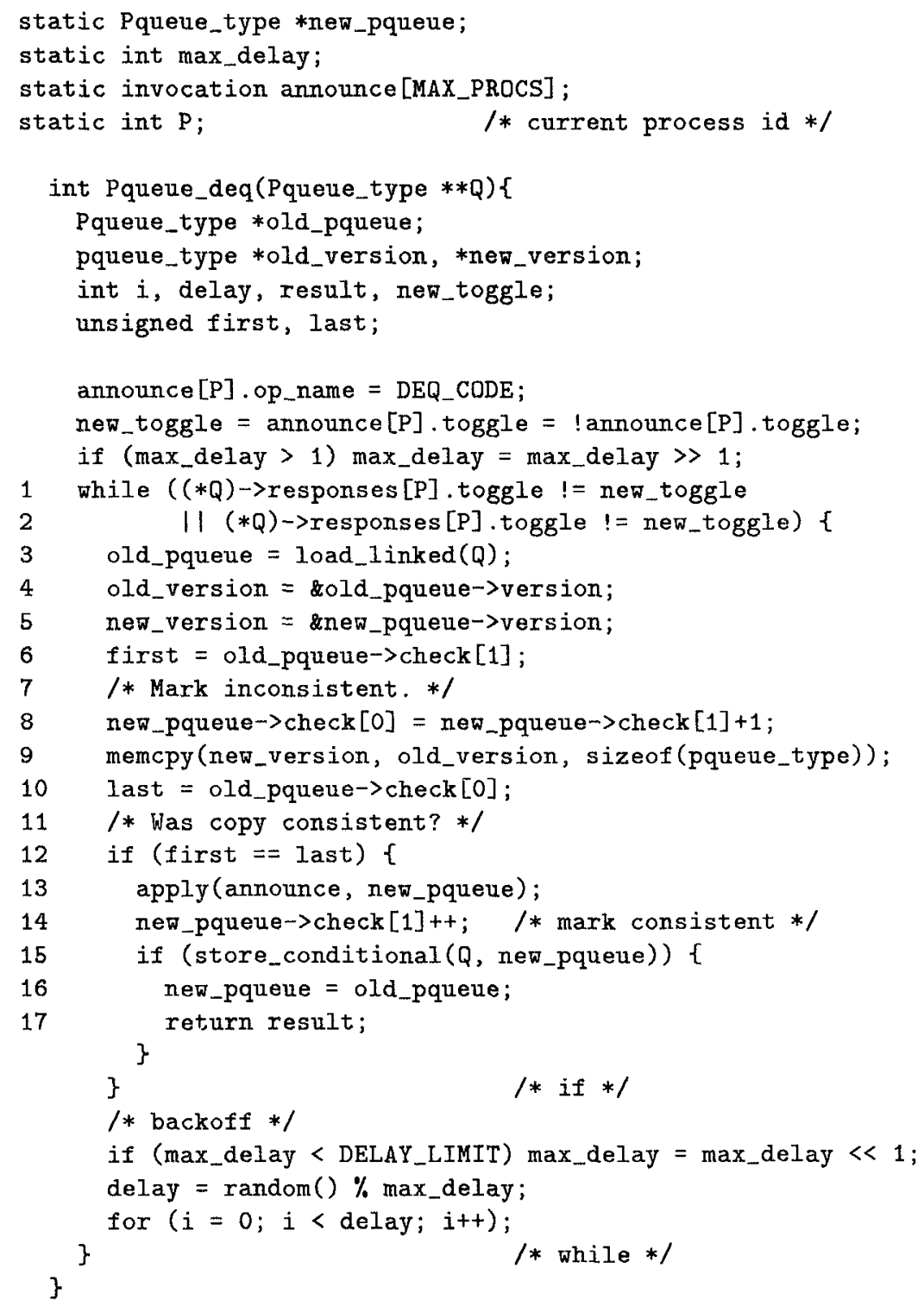

Fig. 10. A wait-free dequeue operation.

either comparison fails, the operation is still incomplete. This comparison must be done twice to avoid the following race condition: (1) $P$ reads a pointer to version $v$. (2) $Q$ replaces $v$ with $v^{\prime}$. (3) $Q$ starts another operation, scans announce, applies $P$ 's operation to the new value of $v$, and stores the tentative result in $v$ 's response array. (4) $P$ observes that the toggle bits match and returns. (5) $Q$ fails to install $v$ as the next version, ensuring that $P$ has returned the wrong result. 
This protocol guarantees that as long as store_conditional has no spurious failures, each operation will complete after at most two loop iterations. ${ }^{10}$ If $P$ 's first or second store_conditional succeeds, the operation is complete. Suppose the first store_conditional fails because process $Q$ executed an earlier store_conditional, and the second store_conditional fails because process $Q^{\prime}$ executed an earlier store_conditional. $Q^{\prime}$ must have scanned the announce array after $Q$ performed its store_conditional, but $Q$ performed its store_conditional after $P$ updated its invocation structure, and therefore $Q^{\prime}$ must have carried out $P^{\prime}$ s operation and set the toggle bits to agree. The process applies the termination test repeatedly during any backoff.

We are now ready to explain why sequential operations must be total. Notice that in the benchmark program (Figure 3), each process enqueues an item before dequeuing. One might assume, therefore, that no dequeue operation will ever observe an empty queue. This assumption is wrong. Each process reads the object version and the announce array as two distinct steps, and the two data structures may be mutually inconsistent. A slow process executing a dequeue might observe an empty queue, and then observe an announce array in which dequeue operations outnumber enqueue operations. This process's subsequent store_conditional will fail, but not until the sequential dequeue operation has been applied to an empty queue. This issue does not arise in the lock-free protocol.

Figure 11 shows the time needed to complete the benchmark program for the wait-free protocol. The throughput increases along with concurrency because the amount of copying per operation is reduced. Nevertheless, there is a substantial overhead imposed by scanning the announce array, and, more importantly, copying the version's responses array with each operation. As a practical matter, the probabilistic guarantee against starvation provided by exponential backoff appears preferable to the deterministic guarantee provided by operation combining.

\section{LARGE OBJECTS}

In this section, we show how to extend the previous section's protocols to objects that are too large to be copied all at once. For large objects, copying is likely to be the major performance bottleneck. Our basic premise is that copying should therefore be under the explicit control of the programmer, since the programmer is in a position to exploit the semantics of the application.

A large object is represented by a set of blocks linked by pointers. Sequential operations of large objects are written in a functional style: an operation that changes the object's state does not modify the object in place. Instead, it constructs and returns a logically distinct version of the object. By logically distinct, we mean that the old and new versions may in fact share a substantial amount of memory. It is the programmer's responsibility

\footnotetext{
${ }^{10}$ Because spurious fallures are possible, this loop requires an explicit termination test; it cannot simply count to two.
}

ACM Transactions on Programming Languages and Systems, Vol. 15, No 5, November 1993 


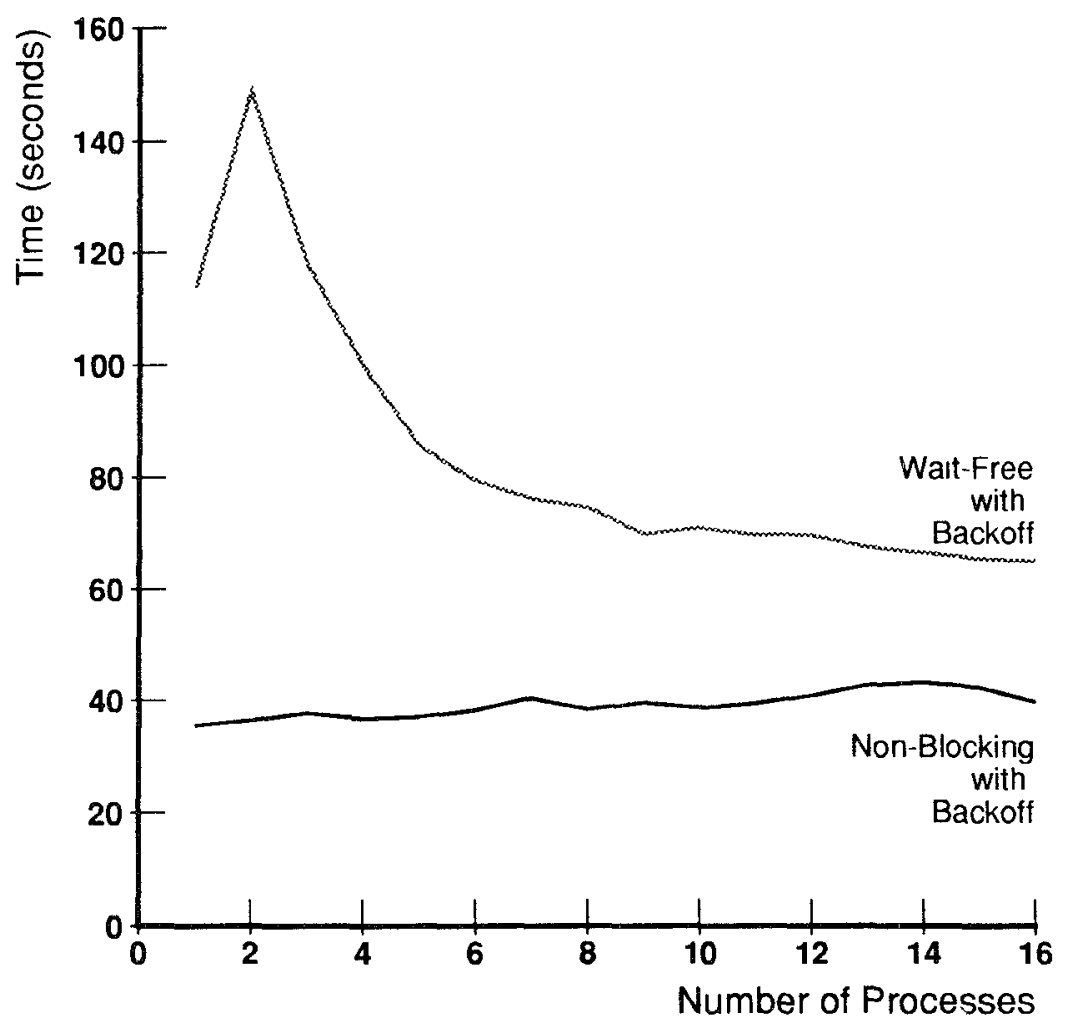

Fig, 11. Lock-free versus wait-free.

to choose a sequential implementation that performs as little copying as possible.

The basic technique is the following. Each process (1) reads the pointer using load_linked, (2) applies the sequential operation, which returns a pointer to a new version, and (3) calls store_conditional to swing the pointer from the old version to the new.

Memory management is slightly more complex. Since an operation may require allocating multiple blocks of memory, each process owns its own pool of blocks. When a process creates a new version of the object, it explicitly allocates new blocks by calling alloc, and it explicitly frees old blocks by calling free. The copy primitive copies the contents of one block to another. If the attempt succeeds, the process acquires ownership of the blocks it freed and relinquishes ownership of the blocks it allocated.

A process keeps track of its blocks with a data structure called a recoverable set (set_type). The abstract state of a recoverable set is given by three sets of blocks: committed, allocated, and freed. The set_free operation inserts a block in freed, and set_alloc moves a block from committed to allocated and returns its address. As shown in Figure 12, alloc calls set_alloc and marks the resulting block as inconsistent, while free simply calls set_free. 


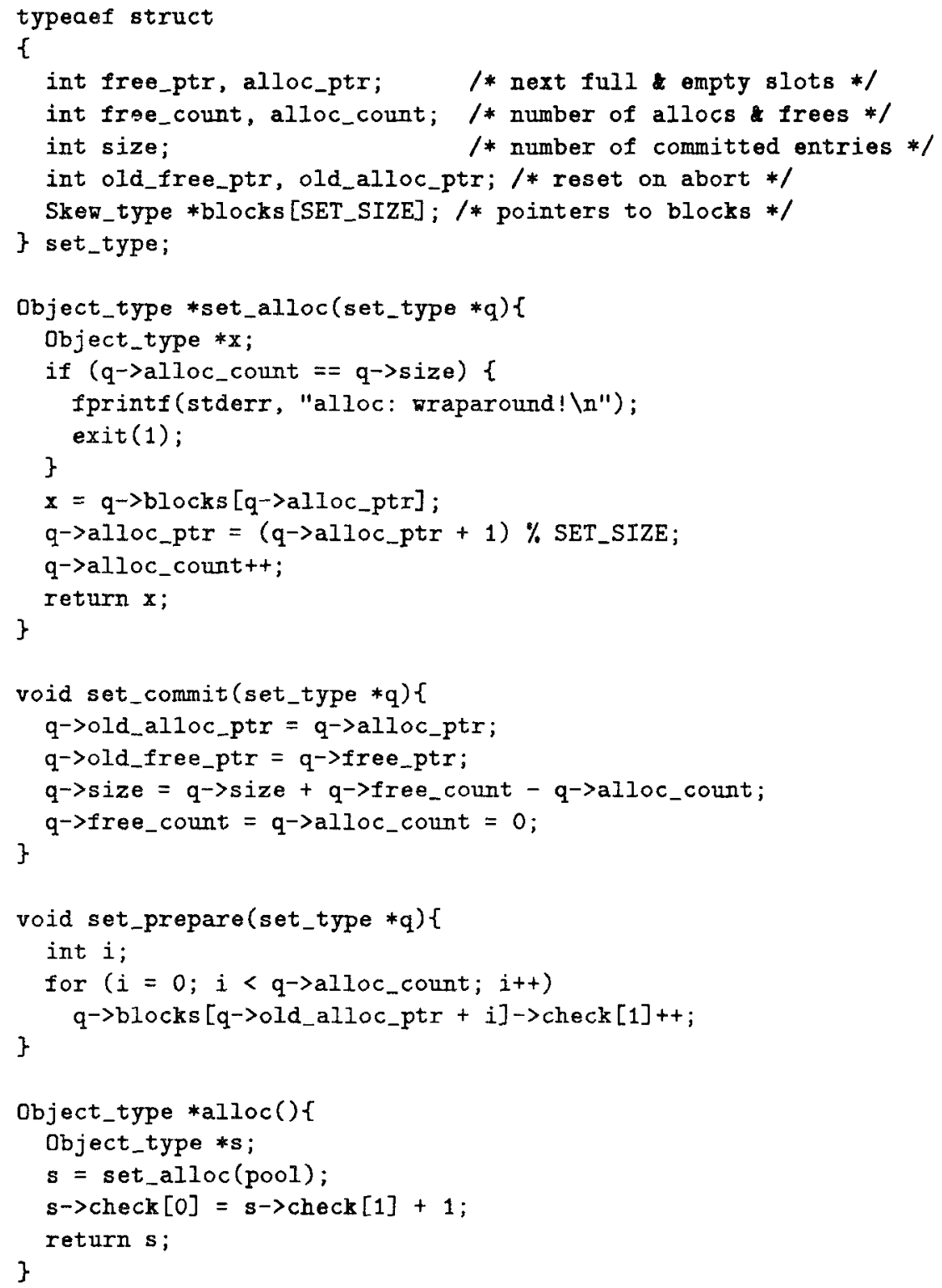

Fig. 12. Part of a recoverable set implementation.

The recoverable set type provides three additional operations, not explicitly called by the programmer. Before executing the store_conditional, the process calls set_prepare to mark the blocks in allocated as consistent. If the store_conditional succeeds, it calls set_commit to set committed to the union of freed and committed, and if it fails, it calls set_abort to set both freed and allocated to the empty set.

ACM Transactions on Programming Languages and Systems, Vol. 15, No. 5, November 1993. 
It might also be necessary for processes to share a pool of blocks. If a process exhausts its local pool, it can allocate multiple blocks from the shared pool, and if it acquires too many blocks, it can return the surplus to the shared pool. The shared pool should be accessed as infrequently as possible, since otherwise it risks becoming a contention "hot spot." Some techniques for implementing shared pools appear elsewhere [Herlihy 1990]; we did not use a shared pool in the prototypes shown here.

As in the small object protocol, a process checks for consistency whenever it copies a block. If the copy is inconsistent, the process transfers control back to the main loop (e.g., using the Unix longjmp).

\subsection{Experimental Results}

For the examples presented in this section, it is convenient to follow some syntactic conventions. Because $\mathrm{C}$ procedures can return only one result value, we follow the convention that all sequential operations return a pointer to a result_type structure containing a value field (e.g., the result of a dequeue) and a version field (the new state of the object). Instead of treating the sequential and concurrent objects as distinct data structures, it is convenient to treat the check array as an additional field of the sequential object, one that is invisible to the sequential operation.

A skew heap [Sleator and Tarjan 1983] is an approximately-balanced binary tree in which each node stores an item, and each node's item is less than or equal to any item in the subtree rooted at that node. A skew heap implements a priority queue, and the amortized cost of enqueuing and dequeuing items in a skew heap is logarithmic in the size of the tree. For our purposes, the advantage of a skew heap over the conventional heap is that update operations leave most of the tree nodes untouched.

The skew_meld operation (Figure 13) merges two heaps. It chooses the heap with the lesser root, swaps its right and left children (for balance), and then melds the right child with the other heap. To insert item $x$ in $h$, skew_enq melds $h$ with the heap containing $x$ alone. To remove an item from $h$, skew_deq (Figure 14) removes the item at the root and melds the root's left and right subtrees.

We modified the priority queue benchmark of Figure 3 to initialize the priority queue to hold 512 randomly generated integers.

Figure 15 shows the relative throughput of a lock-free skew heap, a spin-lock heap with updates in place, and a spin-lock skew heap with updates in place. The lock-free skew heap and the spin-lock heap are about the same, and the spin-lock skew heap has almost twice the throughput of the lock-free skew heap, in agreement with our experimental results for the small object protocol.

\section{CONCLUSIONS}

Conventional concurrency control techniques based on mutual exclusion were originally developed for single-processor machines in which the processor was multiplexed among a number of processes. To maximize throughput in a 


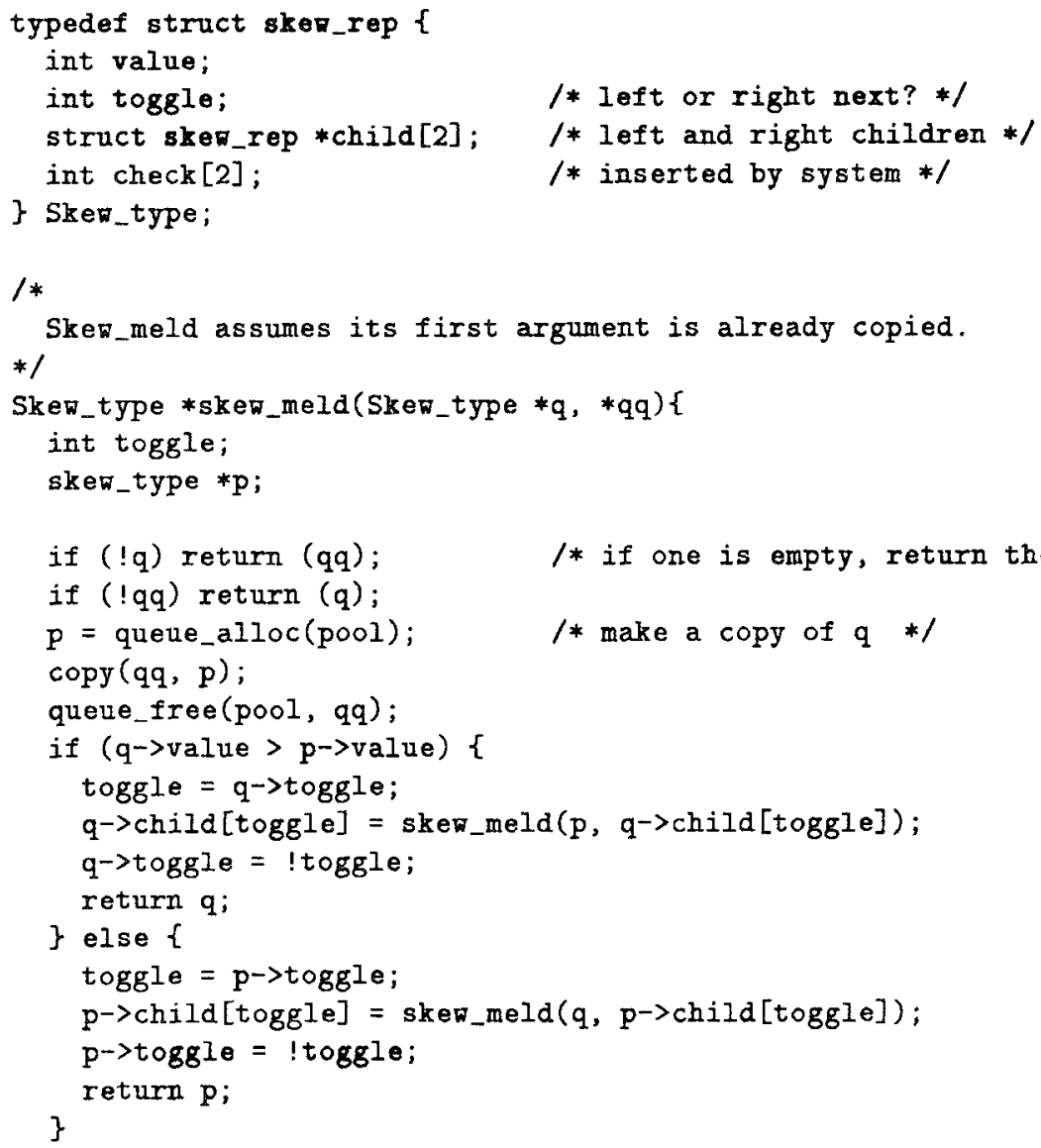

Fig. 13. Skew heap: The meld operation.

uniprocessor architecture, it suffices to keep the processor busy. In a multiprocessor architecture, however, maximizing throughput is more complex. Individual processors are often subject to unpredictable delays, and throughput will suffer if a process capable of making progress is unnecessarily forced to wait for one that is not.

To address this problem, a number of researchers have investigated waitfree and lock-free algorithms and data structures that do not rely on waiting for synchronization. Much of this work has been theoretical. There are two obstacles to making such an approach practical: conceptual complexity, and performance. Conceptual complexity refers to the well-known difficulty of reasoning about the behavior of concurrent programs. Any practical methodology for constructing highly-concurrent data structures must include some mechanism for ensuring their correctness. Performance refers to the observation that avoiding waiting, like most other kinds of fault-tolerance, incurs a 


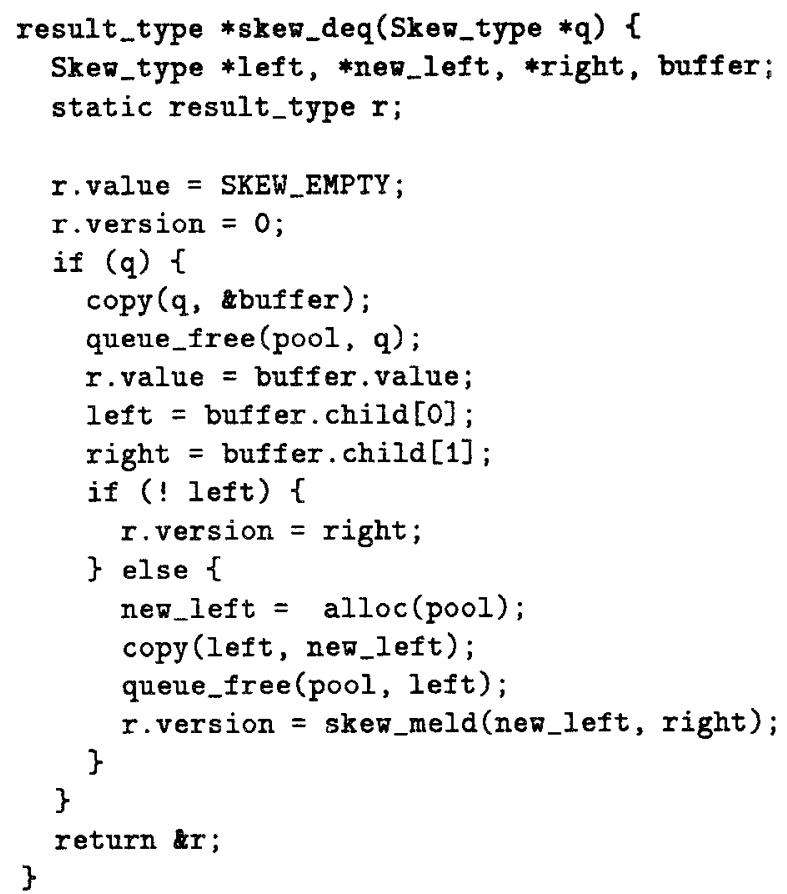

Fig. 14. Skew heap: The dequeue operation.

cost when it is not needed. For a methodology to be practical, this overhead must be kept to a minimum.

In the methodology proposed here, we address the issue of conceptual complexity by proposing that programmers design their data structures in a stylized sequential manner. Because these programs are sequential, both formal and informal reasoning are greatly simplified.

We address the issue of performance in several ways.

-We observe that the load_linked and store_conditional synchronization primitives permit significantly simpler and more efficient algorithms than compare \& swap.

-We propose simple and efficient memory management techniques.

-We provide experimental evidence that a naive implementation of a lockfree protocol incurs unacceptable memory contention, but that this contention can be alleviated by applying well-known techniques such as exponential backoff. Our prototype implementations (using inefficient simulated synchronization primitives) outperform conventional ("test-andtest-and-set") spin-lock implementations, and lie within a factor of two or more sophisticated (exponential backoff) spin-lock implementations.

-For large objects, programmers are free to exercise their ingenuity to keep the cost of copying under control. Whenever possible, correctness should be the responsibility of the system, and performance the responsibility of the programmer. 


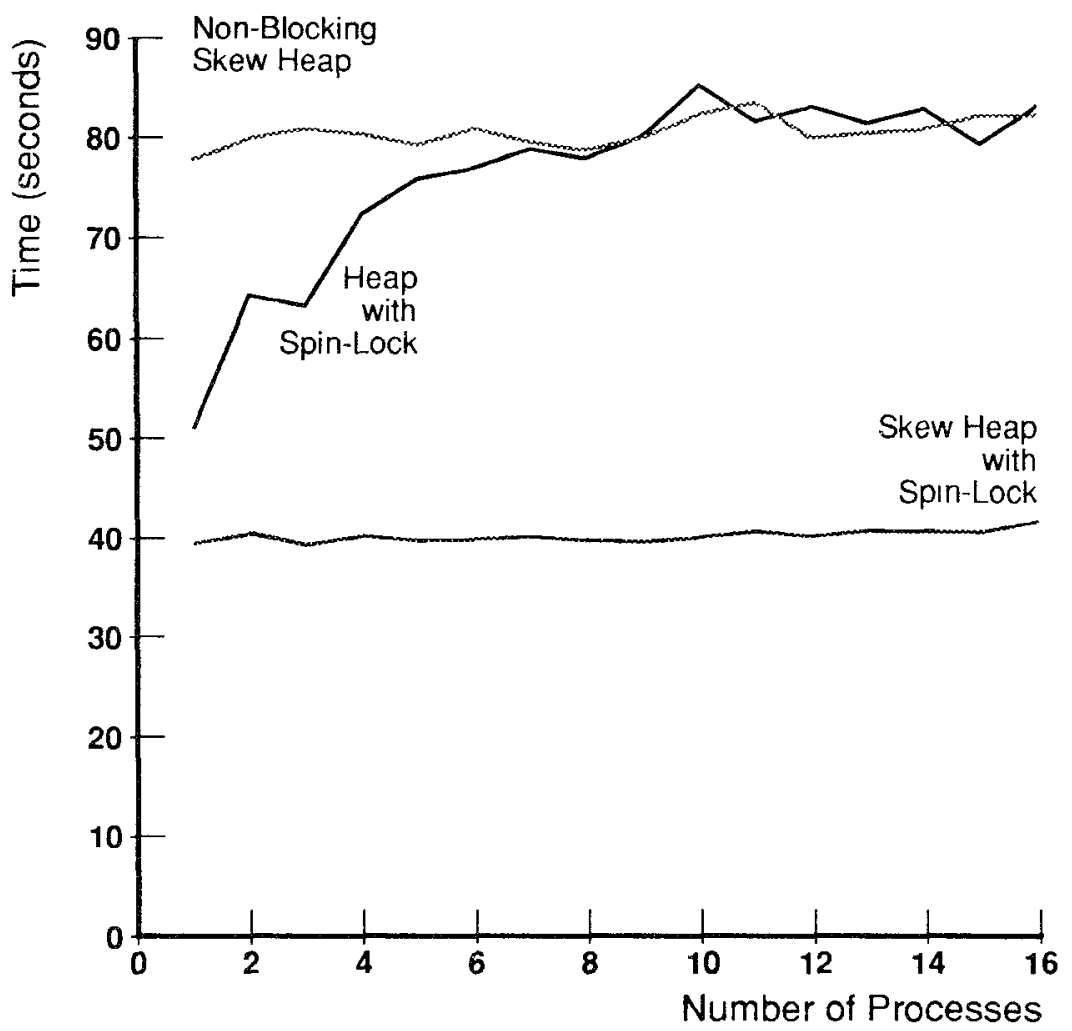

Fig. 15. Large heap throughput.

A promising area for future research concerns how one might exploit type-specific properties to increase concurrency. Any such approach would have to sacrifice some of the simplicity of our methodology, since the programmer would have to reason explicitly about concurrency. Nevertheless, perhaps one could use our methodology to construct simple concurrent objects that could be combined to implement more complex concurrent objects, in the same way that B-link [Lehman and Yao 1981] trees combine a sequence of low-level atomic operations to implement a single atomic operation at the abstract level.

As illustrated by Andrews and Schneider's comprehensive survey [Andrews and Schneider 1983], most language constructs for shared memory architectures focus on techniques for managing mutual exclusion. Because the transformations described here are simple enough to be performed by a compiler or preprocessor, it is intriguing to speculate about a programming language that might support the methodology proposed here. For example, inheritance might be a convenient way to combine the object fields (e.g., check variables) used by the run-time system with those introduced by the programmer. Programming language design raises many complex issues that lie well beyond the scope of this paper, but the issue merits further attention. 


\section{ACKNOWLEDGMENTS}

I would like to thank Brian Bershad, Abdelsalam Heddaya, Narayanan Krishnakumar, Butler Lampson, Michael Merritt, William O'Farrell, Edward Segall, Himanshu Sinha, Peter Sweeney, Gadi Taubenfeld, Bill Weihl, and the anonymous referees for comments on this and earlier versions of this work.

\section{REFERENCES}

Allemany, J., AND FeltoN, E. W. 1992. Performance issues in non-blocking synchronization on shared memory multiprocessors. In Proceedings of the 11th Annual ACM Symposium on Principles of Distrubuted Computing (Aug.). ACM, New York, pp. 125-134.

ANDERson, T. E. 1990. The performance of spin lock alternatives for shared-memory multiprocessors. IEEE Trans. Parallel Distrib. Syst. 1, 1 (Jan.) 6-16.

Andrews, G. R., AND Schnelder, F. B. 1983. Concepts and notations for concurrent programming. ACM Comput. Surv. 15, 1, 3-43.

BAyER, R., AND SCHKolnick, M. 1977. Concurrency of operations on B-trees Acta Inf. 1, 1, $1-21$.

Biswas, J., AND Browne, J. C. 1987. Simultaneous update of priority structures. In Proceedings of the 1987 International Conference on Parallel Processing. pp 124-131.

Burns, J. E., AND Pererson, G. L. 1987. Constructing multi-reader atomic values from non-atomic values. In Proceedings of the 6th ACM Symposium on Principles of Distrubuted Computing. ACM, New York, pp. 222-231.

Chor, B., Israeli, A., AND LI, M. 1987. On processor coordination using asynchronous hardware. In Proceedings of the 6th ACM Symposium on Principles of Distributed Computing. ACM, New York, pp. 86-97.

Cormen, T. H., Leiserson, C. E., AND Rivest, R. L. 1990. Introduction to Algorithms. MIT Press, Cambridge, MA.

DOlev, D., Dwork, C., AND STOCKMEYER, L. 1987. On the minimal synchronism needed for distributed consensus. J. ACM 34, 1 (Jan.), 77-97.

Dwork, C., LYNCH, N., AND STOCKMEYER, L. 1988. Consensus in the presence of partial synchrony. J. ACM 35, 2 (Apr.), 228-323.

Dwork, C., SHMoys, D., AND STOCKMEYer, I. 1986. Flipping persuasively in constant expected time. In The 27th Annual Symposium on Foundations of Computer Science (Oct.). 222-232.

Eluis, C. S. 1980. Concurrent search and insertion in 2-3 trees. Acto Inf. 14, 1 (Apr.), $63-86$.

Encore Computer Cokporation 1989. Multimax technical summary. Order Number 726-01759, Rev E.

Fischer, M., Lynch, N. A., and Paterson, M. S. 1985. Impossibility of distrubuted commit with one faulty process. J. ACM 32, 2 (Apr.), 374-382.

Ford, R., AND CALHoun, J. 1984. Concurrency control mechanisms and the serializability of concurrent tree algorithms. In 3rd ACM Symposium on Principles of Database Systems. ACM, New York, pp. 51-60.

Gottlieb, A., Grishman, R., Kruskal, C. P., Mcauliffe, K. P., Rudolph, L., And Snir, M. 1984. The nyu ultracomputer-designing an MIMD parallel computer. IEEE Trans. Comput. C-32, 2 (Feb.), 175-189.

Gotrlieb, A., Lubachevsky, B. D., ANd RudolPh, L. 1983. Basic techniques for the efficient coordination of very large numbers of cooperating sequential processors. ACM Trans. Program. Lang. Syst. 5, 2 (Apr.), 164-189.

GUIBAS, L.. AND SEDGEwICK, R. 1978. A dichromatic framework for balanced trees. In 19th ACM Symposium on Foundations of Computer Sctence. ACM, New York, pp. 8-21.

Herlihy, M. P. 1991. Wait-free synchronization. ACM. Trans. Program. Lang. Syst. 13, 1 (Jan.), 123-149. 
Herlity, M. P. 1990. A methodology for implementing highly concurrent data structures. In Proceedings of the 2nd ACM SIGPLAN Symposium on Principles and Practice of Parallel Programming (Mar). ACM, New York, pp. 197-206.

Herlihy, M, AND Moss. J. E. B. 1993. Transactional memory: Architectural support for lock-free data structures. In the 1993 20th Annual Symposium on Computer Architecture (San Diego, Calif., May) pp. 289-301.

Herlihy, M., aNd WING, J. 1987 Axioms for concurrent objects. In 14th ACM Symposium on Principles of Programming Languages (Jan.). ACM, New York, pp. 13-26.

IBM. System /370 principles of operation Order Number GA22-7000.

Jensen, E. H, Hagensen, G W., And Broughton, J M. 1987. A new approach to exclusive data access in shared memory multiprocessors. Tech. Rep. UCRL-97663, Lawrence Livermore National Laboratory, Nov.

JoNES, D. W. 1989. Concurrent operations on priority queues. Commun. ACM 32, 1 (Jan.), $132-137$.

KANE, G. 1989. MIPS RISC Architecture. Prentice-Hall, Englewood Cliffs, N.J.

Kernighan, B. W. And Ritchie, D. M. 1988 The C Programming Language. Prentice-Hall, Englewood Cliffs, N.J.

Kung, H. T., AND RoBinson, J. T. 1981. On optimistic methods for concurrency control. ACM Trans. Database Syst. 2, 6 (June), 213-226.

LAMPORT, L. 1986. On interprocess communications, parts i and ii. Distrib. Comput. 1, 1 (Apr.), 77-101.

LAMPORT, L. 1983. Specifying concurrent program modules. ACM Trans Program. Lang. Syst 5, 2 (Apr.), 190-222.

LAMPORT, L 1979. How to make a multiprocessor computer that correctly executes multiprocess programs. IEEE Trans. Comput. C-28, 9 (Sept) ), 690

LANIN, V., AND SHASHA, D. 1988 Concurrent set manipulation without locking. In Proceedings of the 7th ACM Symposium on Principles of Database Systems (Mar ). ACM, New York, pp. $211-220$.

Lehman, P. L.. AND YAO, S. B. 1981. Efficient locking for concurrent operations on B-trees. ACM Trans. Database Syst. 6, 4 (Dec.), 650-670.

LI, M., Tromp, J., AND VITÁNYI, P. M. 1991. How to share concurrent wait-free variables. Tech. Rep. CT-91-02, Univ. of Amsterdam, Amsterdam, Netherlands, Mar.

Mellor-CrummeY, J. M., AND SCOTT, M. L 1990. Algorithms for sealable synchronization on shared-memory multiprocessors. Tech. Rep. 342, Unuversity of Rochester, Rochester. N.Y.

METCALFE, R., AND BOGGS, D. 1976. Ethernet: Distributed packet switching for local computer networks. Commun. ACM 19.7 (July), 395-404.

Papadimitriou, C. H. 1979. The serializability of concurrent database updates. J. ACM 26, 4 (Oct.), 631-653.

Peterson, G. L. 1983. Concurrent reading while writing. ACM Trans. Program. Lang Syst. 5, 1 (Jan.), 46-55.

Peterson, G. L., And Burns, J. E. 1986. Concurrent reading whlle writing il: The multi-writer case. Tech. Rep. GIT-ICS-86/26, Georgia Institute of Technology, Dec.

Plotkin, S. A. 1989. Sticky bits and universality of consensus. In Proceedings of the 8th ACM Symposium on Principles of Distributed Computing. ACM, New York, pp. 159-176.

RudolPH, L., AND SEgALl, Z. 1983. Dynamic decentralized cache schemes for MIMD parallel processors. In the 11th Annual International Symposium on Computer Architecture (June). pp. 340-347.

SiTES, R. L. 1992. Alpha Architecture Reference Manual. Digital Press, Burlington, Mass.

SleATOR, D. D , AND TARJAN, R. E. 1983. Self adjusting binary trees. In Proceedings of the 15th ACM Symposium on Theory of Computing. ACM, New York, pp. 52-59.

Received November 1991; revised December 1992; accepted December 1992 